\title{
On Generalized $h$-Vectors of Rational Polytopes with a Symmetry of Prime Order
}

\author{
A. A'Campo-Neuen \\ Fakultät für Mathematik und Informatik, Universität Konstanz, \\ Postfach 5560, D-78457 Konstanz, Germany \\ Annette.ACampo@uni-konstanz.de
}

\begin{abstract}
We prove tight lower bounds for the coefficients of the generalized $h$-vector of a rational polytope with a symmetry of prime order that is fixed-point free on the boundary. These bounds generalize results of Stanley and Adin for the $h$-vector of a simplicial rational polytope with a central symmetry or a symmetry of prime order, respectively.
\end{abstract}

\section{Introduction}

For simplicial polytopes, there is a beautiful complete characterization of the occurring $h$-vectors, conjectured by McMullen and proved by Billera, Lee, Stanley, and McMullen (see [BL], [St1], and [McM]). Assuming that in addition the polytope admits a symmetry, it is natural to ask for the resulting restrictions upon the corresponding $h$-vector.

Björner conjectured tight lower bounds for the coefficients of the $h$-vector of a centrally symmetric simplicial polytope that were proved by Stanley in [St2], using the theory of toric varieties. After a small perturbation preserving the combinatorics as well as the central symmetry one can assume that the polytope is rational. Then the polytope defines a rationally smooth projective toric variety with an equivariant involution and the coefficients of the $h$-vector are the Betti numbers of the variety. Stanley in fact proves lower bounds for those Betti numbers.

Stanley's results were generalized by Adin to the case of rational simplicial polytopes admitting a symmetry of prime order without fixed points on the boundary of the polytope (see [Ad]). Here it is essential to assume that the polytope is rational, and Adin's statement does not make sense without it.

The aim of this paper is to prove an analogous result to Adin's for rational polytopes that are no longer assumed to be simplicial. Then the Betti numbers of the associated projective toric varieties in general are not combinatorial invariants. We have to replace 
singular cohomology by rational intersection cohomology of middle perversity to arrive at combinatorial invariants. The intersection Betti numbers can be expressed in terms of numbers of certain flags of faces of the polytope and form the so-called generalized $h$-vector (see [St3]).

We obtain lower bounds for the coefficients of the generalized $h$-vector of a rational polytope with the same type of symmetry as studied by Adin. In his proof Adin uses the refined Poincaré series of the Stanley-Reisner ring associated to the polytope. We generalize this method by considering the refined Poincaré series of the equivariant intersection cohomology instead. Note that in the simplicial case the equivariant intersection cohomology forms a ring which is isomorphic to the Stanley-Reisner ring.

In Section 1 we fix the notation, recall Adin's result, and state our generalization (see Theorem 1.2). Section 2 is devoted to the interpretation of the result in terms of projective toric varieties (see Theorem 2.1). Section 3 contains the facts about equivariant intersection cohomology that are needed to complete the proof which is carried out in Section 4.

\section{Rational Polytopes with a Symmetry of Prime Order}

Let $P$ denote a rational polytope in $\mathbb{R}^{n}$, i.e., the convex hull of a finite number of points with rational coordinates. We assume that $P$ is of full dimension and that the origin is placed in its center of mass. By definition, a symmetry of $P$ is a bijection of $P$ induced by a linear map $A$. Note that the assumption that $P$ is rational implies $A \in \mathrm{GL}_{n}(\mathbb{Q})$.

A symmetry of $P$ does not have fixed points on the boundary of the polytope if and only if the inducing linear map $A$ does not have 1 as an eigenvalue. This means that the linear map permutes the proper faces of the polytope without mapping any face onto itself.

We denote the set of proper faces of $P$ by $\mathcal{F}(P)$. (For technical reasons, we also consider the empty set as a face with the convention $\operatorname{dim} \emptyset=-1$.) If $P$ admits a symmetry of prime order $p$ without fixed points on the boundary, then all the orbits of the induced permutation of $\mathcal{F}(P)$ have length $p$, except the orbit of the empty face. So in particular, the numbers $f_{j}$ of faces of dimension $j$ of $P$ (for $0 \leq j \leq n-1$ ) are all divisible by $p$. Moreover, the dimension $n$ is a multiple of $p-1$, since the linear map $A \in \mathrm{GL}_{n}(\mathbb{Q})$ defining the symmetry does not have 1 as an eigenvalue, and therefore its characteristic polynomial is a power of the $p$ th cyclotomic polynomial $1+x+\cdots+x^{p-1}$.

For example, the $(p-1)$-simplex $S \subset \mathbb{R}^{p-1}$, obtained as the convex hull of the canonical basis vectors $e_{1}, \ldots, e_{p-1}$ and the vector $v:=-\sum_{i=1}^{p-1} e_{i}$, has a symmetry of order $p$, induced by the linear map, sending $e_{i}$ to $e_{i+1}$ for all $i<p-1$ and $e_{p-1}$ to $v$. Polytopes admitting a symmetry of order 2 without fixed points on the boundary are precisely centrally symmetric polytopes.

The $h$-vector $\left(h_{0}, \ldots, h_{n}\right)$ of an $n$-dimensional polytope $P$ is defined as follows:

$$
\sum_{j=0}^{n} h_{j} x^{j}:=\sum_{j=0}^{n} f_{j-1}(x-1)^{n-j} \in \mathbb{Z}[x],
$$

where $f_{j}$ denotes the number of $j$-dimensional faces of $P$ (for $-1 \leq j \leq n-1$ ). For 
example, $h_{n}=1, h_{n-1}=f_{0}-n$, and $h_{0}=\sum_{j=0}^{n}(-1)^{n-j} f_{j-1}$. If $P$ is a simplex, then $h_{j}=1$ for all $j$. Note that the definition of the $h$-vector makes sense for arbitrary polytopes. As we mentioned in the Introduction, if $P$ is rational and simplicial, then the coefficients of the $h$-vector have a topological interpretation as Betti numbers.

Recall that a polynomial $q(x)=\sum_{i=0}^{n} a_{i} x^{i} \in \mathbb{Z}[x]$ is called symmetric if $a_{i}=a_{n-i}$ for all $i$, and is called unimodal if its coefficients increase up to a certain index and then decrease again.

In [Ad], Adin proved the following theorem, thereby generalizing Stanley's result [St2] for centrally symmetric simplicial polytopes:

Theorem 1.1 [Ad]. Let $\left(h_{0}, \ldots, h_{n}\right)$ denote the $h$-vector of a rational simplicial polytope $P$ with a symmetry of prime order $p$ that is fixed-point free on the boundary. Then $p-1$ divides $n$, and the polynomial

$$
\sum_{j=0}^{n} h_{j} x^{j}-\left(1+x+\cdots+x^{p-1}\right)^{r} \in \mathbb{Z}[x], \quad \text { where } \quad r:=\frac{n}{p-1},
$$

is symmetric and unimodal, and all its coefficients are divisible by $p$.

Since $h_{0}=1$, the constant term of the polynomial is zero. So the unimodality of the polynomial implies in particular that the coefficients are nonnegative. The resulting lower bounds for the $h_{j}$ are tight, as Adin shows by constructing examples of rational simplicial polytopes with a "minimal" $h$-vector. More precisely, given a natural number $n$ and a prime number $p$ such that $p-1$ divides $n$, and, for $i=1, \ldots, r:=n /(p-1)$, a copy $S_{i}$ of the $(p-1)$-simplex $S$ in $V_{i}:=\mathbb{R}^{p-1}$, then the convex hull of the union $\bigcup_{i=1}^{r} S_{i}$ in the direct sum $\bigoplus_{i=1}^{r} V_{i}$ has a symmetry of order $p$ that is fixed-point free on the boundary. In this case $\sum_{j=0}^{n} h_{j} x^{j}=\left(1+x+\cdots+x^{p-1}\right)^{r}$.

In this article we want to generalize Adin's result further. Namely, we want to consider rational polytopes that are not necessarily simplicial. Following Stanley (see [St3]), we introduce two polynomials $h_{P}$ and $g_{P}$ for each polytope $P$, that are defined by recursion over the set of faces of $P$ as follows:

(i) $g_{\emptyset} \equiv 1$,

(ii) $h_{P}(x)=\sum_{F \in \mathcal{F}(P)}(x-1)^{\operatorname{dim} P-\operatorname{dim} F-1} g_{F}(x)$,

(iii) $g_{P}(x)=\tau_{\leq[(\operatorname{dim} Q) / 2]}\left((1-x) h_{P}(x)\right)$,

where $\tau_{\leq r}$ denotes the truncation operator $\tau_{\leq r}\left(\sum_{i=0}^{n} a_{i} x^{i}\right):=\sum_{i=0}^{r} a_{i} x^{i}$.

The vector formed by the coefficients of the polynomial $h_{P}$ is called the generalized $h$-vector of $P$. Note that $h_{P}$ depends only on the set of faces $\mathcal{F}(P)$ as a partially ordered set, and it involves not only the face numbers but also the numbers of certain flags of faces of $P$. If $P$ is simplicial, then the generalized and the ordinary $h$-vector coincide. However, this is not true for a general rational polytope. For example, the ordinary $h$ vector of the three-dimensional cube is $(1,-1,5,1)$ whereas the generalized $h$-vector is $(1,5,5,1)$.

The aim of this paper is to prove the following generalization of Theorem 1.1:

Theorem 1.2. Let $P$ be a rational polytope of dimension $n$ admitting a symmetry of 
prime order $p$ without fixed points on the boundary, and let $\left(\tilde{h}_{0}, \tilde{h}_{1}, \ldots, \tilde{h}_{n}\right)$ denote its generalized h-vector. Then $p-1$ divides $n$, and the polynomial

$$
\sum_{j=0}^{n} \tilde{h}_{j} x^{j}-\left(1+x+\cdots+x^{p-1}\right)^{r} \in \mathbb{Z}[x], \quad \text { where } \quad r:=\frac{n}{p-1},
$$

is symmetric and unimodal, and all its coefficients are divisible by p. In particular, all the coefficients are nonnegative.

\section{Projective Toric Varieties and Intersection Cohomology}

Both Stanley and Adin prove their results by first translating them to statements about projective toric varieties, and we follow the same strategy. Let $P \subset \mathbb{R}^{n}$ be a rational $n$-dimensional polytope with a symmetry of prime order $p$, induced by a linear map $A \in \mathrm{GL}_{n}(\mathbb{Q})$. Suppose that $A$ does not have 1 as an eigenvalue. Then the characteristic polynomial of $A$ equals $\left(1+x+\cdots+x^{p-1}\right)^{r}$ for $r:=n /(p-1)$, and as an $A$-module $\mathbb{Q}^{n}$ decomposes into a direct sum of $r$ copies of $\mathbb{Q}[t] /\left(1+x+\cdots+x^{p-1}\right)$. So, in particular, the rational canonical form of $A$ has only integer entries. Therefore after a suitable rational base change we can assume that $A \in \mathrm{GL}_{n}(\mathbb{Z})$.

The polytope $P$ defines a fan $\Delta_{P}:=\left\{\mathbb{R}_{\geq 0} \cdot(F \cup\{0\}) \mid F \in \mathcal{F}(P)\right\}$ in $\mathbb{R}^{n}$ consisting of all the cones through proper faces of $P$. Since $P$ is rational all the cones are rational with respect to the lattice $\mathbb{Z}^{n}$. The pair $\left(\Delta_{P}, \mathbb{Z}^{n}\right)$ corresponds to an $n$-dimensional projective toric variety $X=X_{P}$ (see, e.g., [Fu]). The vector space $\mathbb{R}^{n}$ is naturally identified with the Lie algebra of the maximal compact subgroup $S$ of the algebraic torus $T$ acting on $X$, and the lattice $\mathbb{Z}^{n}$ in $\mathbb{R}^{n}$ is the kernel $N$ of the exponential map from $\operatorname{Lie}(S)$ to $S$.

Moreover, the linear map $A \in \mathrm{GL}_{n}(\mathbb{Z})$ defines a unique automorphism $\varphi_{A}$ of $T$ determined by the property $\left.\left(d \varphi_{A}\right)_{e}\right|_{N}=A$. The isomorphism $\varphi_{A}$ extends to an automorphism of the toric variety $X_{P}$ since $A$ permutes the cones of $\Delta_{P}$ (for details on equivariant morphisms of toric varieties see, e.g., $[\mathrm{Fu}])$. The assumption that $A$ has no fixed points on the boundary of $P$ implies that the only $\varphi_{A}$-stable $T$-orbit in $X_{P}$ is the dense orbit.

Conversely, every projective toric variety $X$ with a $T$-equivariant automorphism $\varphi$ of prime order $p$ can be written in the form $X=X_{P}$ for some polytope $P$ such that the linear map $A=\left.d \varphi_{e}\right|_{N} \in \mathrm{GL}_{n}(\mathbb{Z})$ corresponding to $\varphi$ induces a symmetry of $P$. If in addition $\varphi$ fixes only the dense $T$-orbit, then $A$ does not have 1 as an eigenvalue.

If $P$ is simplicial, then the associated projective toric variety is rationally smooth, i.e., it has at worst finite quotient singularities. In that case the odd Betti numbers of singular cohomology vanish, and the even Betti numbers are precisely the entries of the $h$-vector of $P$, i.e., $b^{2 j}\left(X_{P}\right)=h_{j}$ for $0 \leq j \leq n$. So the Poincare polynomial of the toric variety $X_{P}$ is of the form

$$
P_{X}(t):=\sum_{j=0}^{2 n} \operatorname{dim}\left(H^{j}(X ; \mathbb{Q})\right) t^{j}=\sum_{j=0}^{n} h_{j} t^{2 j} .
$$

By Poincaré duality, the polynomial $P_{X}$ is symmetric, and in particular $h_{j}=h_{n-j}$ for all $j$. These relations for the coefficients of the $h$-vector are also known as the DehnSommerville equations. Moreover, the polynomial $P_{X}$ is unimodal as a consequence 
of the hard Lefschetz theorem. (The fact that the hard Lefschetz theorem for singular cohomology is true for rationally smooth varieties follows from the fact that there is also a hard Lefschetz theorem for intersection cohomology of middle perversity of algebraic varieties (see [BBD] and [Sa]). If the variety is rationally smooth, then the intersection cohomology of middle perversity and singular cohomology coincide.)

For example, the $(p-1)$-simplex $S$, for a given prime number $p$, corresponds to the toric variety $\mathbb{P}^{p-1}(\mathbb{C})$ whose Poincaré polynomial is $1+t^{2}+\cdots+t^{2(p-1)}$, and, as stated in Section 1, all the entries of the $h$-vector of $S$ are equal to 1 .

If we drop the assumption that $P$ is simplicial, then the associated projective toric variety $X_{P}$ can have more complicated singularities, and the Betti numbers of singular cohomology are no longer combinatorial invariants. However, instead of singular cohomology, we can consider the rational intersection cohomology of middle perversity. It turns out that the intersection Betti numbers are in fact given by the entries of the generalized $h$-vector $\left(\tilde{h}_{0}, \ldots, \tilde{h}_{n}\right)$ of $P$. More precisely, the odd intersection Betti numbers $I b_{2 j+1}(X)$ vanish and the even intersection Betti numbers satisfy $I b_{2 j}(X)=\tilde{h}_{j}$. (In his survey article [St3], Stanley remarks that this result was proved independently by Bernstein, Khovanskii, and MacPherson, but their proofs have not been published. Proofs appeared in [Fi] by Fieseler and in [DL] by Denef and Loeser.) In particular, the intersection Betti numbers are combinatorial invariants.

The Poincaré polynomial of the intersection cohomology of middle perversity

$$
I P_{X}(t):=\sum_{j=0}^{2 n} \operatorname{dim}\left(I H^{j}(X ; \mathbb{Q})\right) t^{j}=\sum_{j=0}^{2 n} I b_{j} t^{j}
$$

is symmetric, since Poincaré duality holds for intersection cohomology. Moreover, as mentioned above there is also a hard Lefschetz theorem for intersection cohomology of middle perversity and therefore $I P_{X}$ is unimodal (see Corollary 3.2 of [St3]).

So in terms of toric varieties Theorem 1.2 reads as follows:

Theorem 2.1. Let $X$ be a projective toric variety of dimension $n$, and suppose that $X$ has a T-equivariant automorphism $\varphi$ of prime order $p$, defining a fixed-point-free permutation of the $T$-orbits in the boundary of $T$ in $X$. Then $p-1$ divides $n$, and the polynomial

$$
q(x):=\sum_{j=0}^{n} I b_{2 j} x^{j}-\left(1+x+\cdots+x^{p-1}\right)^{r} \in \mathbb{Z}[x], \quad \text { where } \quad r:=\frac{n}{p-1},
$$

is symmetric and unimodal, and all its coefficients are divisible by $p$.

The symmetry of the polynomial $q$ immediately follows from the symmetry of the Poincaré polynomial $I P_{X}$. So to prove the theorem, we only have to show that $q$ is unimodal and that the coefficients are divisible by $p$. Analogously to Adin's proof of Theorem 1.1, we proceed by interpreting $(1 / p) q$ as a polynomial whose coefficients count certain dimensions. In our case, these are the dimensions of the $\chi$-eigenspaces of $\varphi$ on the graded pieces of the rational intersection cohomology of $X$ for some primitive $p$ th root of unity $\chi$. 
The automorphism $\varphi$ induces a linear map on the $j$ th intersection cohomology $I H^{j}(X ; \mathbb{Q})$ of $X$ for $0 \leq j \leq 2 n$. Since $\varphi$ is of order $p$, the possible eigenvalues of the induced endomorphism are the elements of the group $G$ of $p$ th roots of unity. So over the complex numbers, we have the following decomposition:

$$
I H^{j}(X ; \mathbb{Q}) \otimes \mathbb{C}=\bigoplus_{\chi \in G} I H_{\chi}^{j}(X)
$$

where $I H_{\chi}^{j}(X)$ denotes the $\chi$-eigenspace of $\varphi$. The crucial result is the following proposition.

Proposition 2.2. Let $X$ be a projective toric variety of dimension $n$, and suppose that $X$ has a T-equivariant automorphism $\varphi$ of prime order $p$, defining a fixed-point-free permutation of the $T$-orbits in the boundary of $T$ in $X$. Let $r:=n /(p-1)$. Then

$$
P_{\chi}(t):=\sum_{j=0}^{2 n}\left(\operatorname{dim} I H_{\chi}^{j}(X)\right) t^{j}=\frac{1}{p}\left(I P_{X}(t)-\left(1+t^{2}+\cdots+t^{2(p-1)}\right)^{r}\right)
$$

for every primitive $p$ th root of unity $\chi$. In particular, $I P_{X}(t)=\left(1+t^{2}+\cdots+t^{2(p-1)}\right)^{r}$ iff $\varphi$ induces the identity on $\operatorname{IH}^{*}(X ; \mathbb{Q})$.

The proof of this proposition is given in the last section using equivariant intersection cohomology. We conclude this section by deducing Theorem 2.1 from the proposition.

Proof of Theorem 2.1. It suffices to show that $P_{\chi}$ is unimodal. The intersection cohomology of middle perversity $I H^{*}(X ; \mathbb{Q})$ forms a module over the ring of singular cohomology $H^{*}(X ; \mathbb{Q})$. The hard Lefschetz theorem for intersection cohomology asserts that there is an element $\omega \in H^{2}(X ; \mathbb{Q})$ corresponding to a generic hyperplane section of some embedding of $X$ into complex projective space, such that multiplication with $\omega$ induces an injective map from $I H^{2 j}(X ; \mathbb{Q})$ to $I H^{2 j+2}(X ; \mathbb{Q})$ for all $0 \leq j \leq[n / 2]$.

The cohomology class $\omega$ is $\varphi$-invariant since the action of $\varphi$ on $X$ extends to a linear action on the projective space (see the argument given in [St2], provided by Kleiman). Therefore multiplication with $\omega$ commutes with the action of $\varphi$ on the intersection cohomology, and hence $\omega \cdot I H_{\chi}^{2 j}(X) \subset I H_{\chi}^{2 j+2}(X)$ for all $0 \leq j \leq[n / 2]$ and for all characters $\chi$. So, in particular, all the polynomials $P_{\chi}$ are unimodal.

\section{Equivariant Intersection Cohomology}

In this section we first briefly recall some definitions, and then we state the facts that are used in the proof of Proposition 2.2. For the algebraic torus $T=\left(\mathbb{C}^{*}\right)^{n}$, the principal $T$ bundle $E T:=\left(\mathbb{C}^{\infty} \backslash\{0\}\right)^{n} \rightarrow\left(\mathbb{P}^{\infty}(\mathbb{C})\right)^{n}=: B T$ is a classifying bundle since the action of $T$ on the contractible space $E T$ by componentwise scalar multiplication is free. The cohomology ring $H^{*}(B T ; \mathbb{Q})$ of the base space $B T$ is a polynomial ring over $\mathbb{Q}$ in $n$ variables, where each variable has degree 2 , and the corresponding Poincaré series is $\left(1+t^{2}+t^{4}+\cdots\right)^{n}=\left(1-t^{2}\right)^{-n}$. 
Now let $X$ be a toric variety acted on by the torus $T$, and set $E_{q}:=\left(\mathbb{C}^{q+1} \backslash\{0\}\right)^{n}$ for $q \in \mathbb{N} \cup \infty$. The diagonal action of $T$ on $E_{q} \times X$ is free, and forming the quotient by $T$ we obtain $X_{T, q}:=\left(E_{q} \times X\right) / T=E_{q} \times_{T} X$. The natural projection map $p_{q}: X_{T, q} \rightarrow$ $\left(\mathbb{P}^{q}(\mathbb{C})\right)^{n}=: B_{q}$ is a fibration with fiber $X$.

The intersection cohomology group $I H^{j}\left(X_{T, q} ; \mathbb{Q}\right)$ is independent of $q$ for $0 \leq j<$ $2 q$. Therefore it is natural to define equivariant intersection cohomology of $X$ with rational coefficients in the following way (see (2.12) in [Ki]):

$$
I H_{T}^{j}(X ; \mathbb{Q}):=I H^{j}\left(X_{T, q} ; \mathbb{Q}\right) \quad \text { for } \quad j \geq 0, \quad \text { where } \quad q>\frac{j}{2} .
$$

The equivariant intersection cohomology of $X$ is related to its ordinary intersection cohomology in the following way (see, e.g., (2.14) in [Ki]):

Remark. Let $X$ be a projective toric variety. Then

$$
I H_{T}^{*}(X ; \mathbb{Q}) \simeq H^{*}(B T ; \mathbb{Q}) \otimes_{\mathbb{Q}} I H^{*}(X ; \mathbb{Q}) .
$$

This remark can be proved by observing that the spectral sequences associated to the fibrations $p_{q}$ degenerate at the $E_{2}$ level. So for every $j$, there is a filtration of $I H^{j}\left(X_{T, q} ; \mathbb{Q}\right)$ such that the occurring factors are precisely the $E_{2}$ terms of the spectral sequence on the $j$ th diagonal. On the other hand the direct sum of all $E_{2}$ terms equals the tensor product $H^{*}\left(B_{q} ; \mathbb{Q}\right) \otimes_{\mathbb{Q}} I H^{*}(X ; \mathbb{Q})$.

For the Poincare series of the equivariant intersection cohomology $I P_{X}^{T}(t):=$ $\sum_{j=0}^{\infty}\left(\operatorname{dim} I H_{T}^{j}(X ; \mathbb{Q})\right) t^{j}$ we conclude from (3.1) that

$$
I P_{X}^{T}(t)=\left(1-t^{2}\right)^{-n} I P_{X}(t)
$$

We also use the following result, proved by Fieseler in [Fi]:

Proposition 3.1 [Fi]. Let $X$ be a projective toric variety corresponding to the fan $\Delta \in \mathbb{R}^{n}$. Then

$$
I H_{T}^{*}(X ; \mathbb{Q}) \simeq \bigoplus_{\sigma \in \Delta} I H_{T}^{*}\left(U_{\sigma}, U_{\sigma} \backslash B_{\sigma} ; \mathbb{Q}\right),
$$

where, for every cone $\sigma \in \Delta, U_{\sigma}$ denotes the corresponding affine chart of $X$ and $B_{\sigma}$ denotes the corresponding $T$-orbit.

\section{Proof of Proposition 2.2}

Returning to the setting of Theorem 2.1 and Proposition 2.2, we assume that $X$ is a projective toric variety of dimension $n$ with a $T$-equivariant automorphism $\varphi$ of order $p$, for some prime number $p$, and suppose that the only $\varphi$-stable $T$-orbit in $X$ is the dense orbit. Then, as remarked in Section 2, the automorphism $A:=d \varphi_{e} \in \operatorname{Aut}(N)$ of the lattice $N=$ kerexp does not have 1 as an eigenvalue. Since $A$ is of order $p$, the characteristic polynomial of $A$ is a power of the $p$ th cyclotomic polynomial. 
We prove Proposition 2.2 by analyzing the induced action of $\varphi$ on the terms occurring in (3.1), and considering their respective refined Poincaré series.

The refined Poincaré series $P_{W}^{\varphi}$ of a graded complex vector space $W=\bigoplus_{j=0}^{\infty} W^{j}$ with respect to the action of some graded endomorphism $\varphi$ of order $p$ is defined as the following formal power series in one variable over the group ring $\mathbb{Z}[G]$ of the group $G$ of $p$ th roots of unity:

$$
P_{W}^{\varphi}(t):=\sum_{j=0}^{\infty} \sum_{\chi \in G}\left(\operatorname{dim} W_{\chi}^{j}\right) \chi t^{j}
$$

where $W_{\chi}^{j}$ denotes the eigenspace of $\varphi$ in $W^{j}$ for the eigenvalue $\chi \in G$. If, for example, $W$ is a polynomial ring in one variable of degree $d$ which is an eigenvector of $\varphi$ for the eigenvalue $\lambda$, then $P_{W}^{\varphi}(t)=1+\lambda t^{d}+\lambda^{2} t^{2 d}+\cdots=\left(1-\lambda t^{d}\right)^{-1}$.

For $W=I H^{*}(X ; \mathbb{Q}) \otimes \mathbb{C}$ or $I H_{T}^{*}(X ; \mathbb{Q}) \otimes \mathbb{C}$, we denote the corresponding refined Poincaré series by $I P_{X}^{\varphi}$ or $I P_{X}^{T, \varphi}$, respectively. We claim that

$$
I P_{X}^{T, \varphi}(t)=P_{B T}^{\varphi}(t) I P_{X}^{\varphi}(t) .
$$

To justify (4.1) note that the $T$-equivariant automorphism $\varphi$ of $X$ also determines automorphisms of $E_{q}$, as well as of $X_{T, q}$ and $B_{q}$, that commute with the fibrations $p_{q}: X_{T, q} \rightarrow B_{q}$. So $\varphi$ acts naturally on all the terms occurring in the associated spectral sequences. The proof of (3.1) implies that for every $j$ there is an isomorphism between the associated graded $\varphi$-modules of $I H_{T}^{j}(X ; \mathbb{Q})$ and of $\left(H^{*}(B T ; \mathbb{Q}) \otimes_{\mathbb{Q}} I H^{*}(X ; \mathbb{Q})\right)^{j}$. Since $\varphi$ is of finite order and hence semisimple, we can even conclude that $I H_{T}^{j}(X ; \mathbb{Q})$ and $\left(H^{*}(B T ; \mathbb{Q}) \otimes_{\mathbb{Q}} I H^{*}(X ; \mathbb{Q})\right)^{j}$ are isomorphic as $\varphi$-modules and the claim follows.

We now compute $P_{B T}^{\varphi}$. There is a natural identification of $H^{2}(B T ; \mathbb{R})$ with the dual of the Lie algebra $\operatorname{Lie}(S)$ of the maximal compact torus $S \subset T$. On the other hand, $\operatorname{Lie}(S)$ is generated as a real vector space by $N=\operatorname{ker} \exp$ in $\operatorname{Lie}(S)$. So altogether we have a natural isomorphism between $H^{2}(B T ; \mathbb{Q})$ and $N_{\mathbb{Q}}^{\vee}$, and we can view $H^{*}(B T ; \mathbb{Q})$ as the symmetric algebra of $N_{\mathbb{Q}}^{\vee}$.

The action of $\varphi$ on $H^{2}(B T ; \mathbb{Q}) \simeq N_{\mathbb{Q}}^{\vee}$ is given by the dual of $A$. So the eigenvalues are precisely the primitive $p$ th roots of unity, and each $\chi \in G \backslash\{1\}$ occurs with the same multiplicity $r:=n /(p-1)$. Over the complex numbers we can choose a basis of the second cohomology consisting of eigenvectors for $\varphi$, and the cohomology ring of $B T$ is a polynomial ring in those basis vectors. Each $\chi$-eigenvector in the basis of the second cohomology contributes a factor $\left(1-\chi t^{2}\right)^{-1}$ to the refined Poincare series of $B T$, and hence

$$
P_{B T}^{\varphi}(t)=\sum_{j=0}^{\infty} \sum_{\chi \in G}\left(\operatorname{dim} H_{\chi}^{j}(B T)\right) \chi t^{j}=\prod_{1 \neq \chi \in G} \frac{1}{\left(1-\chi t^{2}\right)^{r}} .
$$

To describe the action of $\varphi$ on $I H_{T}^{*}(X ; \mathbb{Q})$ we use that, by Proposition $3.1, I H_{T}^{*}(X ; \mathbb{Q})$ $\simeq \bigoplus_{\sigma \in \Delta} I H_{T}^{*}\left(U_{\sigma}, U_{\sigma} \backslash B_{\sigma} ; \mathbb{Q}\right)$, where $\Delta$ denotes the fan associated to the toric variety $X$. That means that the long exact $I H_{T}^{*}$ sequences associated to the pairs $\left(V_{r}, V_{r-1}\right)$, where $V_{r}:=\bigcup_{\operatorname{dim} \sigma<r} B_{\sigma}$, split into short exact sequences. Since the $\varphi$-action preserves the open exhaustion of $X$ by the open subsets $V_{r}, \varphi$ acts naturally on all the terms occurring in those sequences. Using that $\varphi$ is of finite order, we obtain as above that 
$I H_{T}^{j}(X ; \mathbb{Q})$ and $\bigoplus_{\sigma \in \Delta} I H_{T}^{j}\left(U_{\sigma}, U_{\sigma} \backslash B_{\sigma} ; \mathbb{Q}\right)$ are isomorphic even as $\varphi$-modules. So in particular, the refined Poincaré series of both sides of (3.3) are equal.

The action of $\varphi$ on the right-hand side of (3.3) permutes the direct summands in the same way as the linear map $A$ considered as an element of $\mathrm{GL}_{n}(\mathbb{R})$ permutes the cones of $\Delta$. The only $A$-stable cone of $\Delta$ is the zero cone. We have $B_{0}=T=U_{0}$ and therefore $I H_{T}^{*}\left(U_{0}, U_{0} \backslash B_{0} ; \mathbb{Q}\right)=I H_{T}^{*}(T ; \mathbb{Q})$, which consists of one copy of $\mathbb{Q}$ in degree 0 . So the zero cone only contributes a one-dimensional subspace in the eigenspace for the eigenvalue 1 of $I H_{T}^{0}(X ; \mathbb{Q})$.

Every cone $\sigma \neq 0$ has an orbit of length $p$ under $A$, and for every $j$ the $\varphi$-stable subspace $\bigoplus_{k=1}^{p} I H_{T}^{j}\left(U_{A^{k}(\sigma)}, U_{A^{k}(\sigma)} \backslash B_{A^{k}(\sigma)} ; \mathbb{Q}\right)$ of $I H_{T}^{j}(X ; \mathbb{Q})$ decomposes into a direct sum of copies of the regular representation of the group generated by $\varphi$. That implies that in $\bigoplus_{0 \neq \sigma \in \Delta} I H_{T}^{j}\left(U_{\sigma}, U_{\sigma} \backslash B_{\sigma} ; \mathbb{Q}\right)$ every eigenvalue $\chi \in G$ appears with the same multiplicity.

We obtain

$$
I P_{X}^{T, \varphi}(t)-1=\frac{1}{p} \sum_{\chi \in G}\left(I P_{X}^{T}(t)-1\right) \chi .
$$

Setting $\mu:=(1 / p) \sum_{\chi \in G} \chi \in \mathbb{Z}[G]$ and using (3.2), we arrive at

$$
I P_{X}^{T, \varphi}(t)=\frac{\mu}{\left(1-t^{2}\right)^{n}} I P_{X}(t)+(1-\mu) .
$$

Inserting (4.2) and (4.3) into (4.1) we get

$$
I P_{X}^{\varphi}(t)=\left(\frac{\mu}{\left(1-t^{2}\right)^{n}} I P_{X}(t)+(1-\mu)\right) \prod_{1 \neq \chi \in G}\left(1-\chi t^{2}\right)^{r} .
$$

Lemma 4.1. In the polynomial ring $\mathbb{Z}[G][x]$ over the group ring $\mathbb{Z}[G]$ of the group $G$ of $p$ th roots of unity the following identities hold:

(i) $\mu \prod_{1 \neq \chi \in G}(1-\chi x)^{r}=\mu(1-x)^{n}$;

(ii) $(1-\mu) \prod_{1 \neq \chi \in G}(1-\chi x)^{r}=(1-\mu)\left(1+x+\cdots+x^{p-1}\right)^{r}$, where as above $\mu=(1 / p) \sum_{\chi \in G} \chi$.

Proof. (i) It suffices to check $\mu \chi=\mu$ for all $\chi \in G$. However, this is clear since the multiplication of $\sum_{\chi^{\prime} \in G} \chi^{\prime}$ with an element $\chi$ of $G$ only permutes the summands.

(ii) This part of the lemma is contained in [Ad] as Lemma 13.

Applying Lemma 4.1 and inserting $t^{2}$ for $x$, we can rewrite (4.4) in the following form:

$$
I P_{X}^{\varphi}(t)=\mu I P_{X}(t)+(1-\mu)\left(1+t^{2}+\cdots+t^{2(p-1)}\right)^{r},
$$

which is equivalent to

$$
I P_{X}^{\varphi}(t)=\left(1+t^{2}+\cdots+t^{2(p-1)}\right)^{r}+\frac{1}{p} \sum_{\chi \in G}\left(I P_{X}(t)-\left(1+t^{2}+\cdots+t^{2(p-1)}\right)^{r}\right) \chi .
$$

Now Proposition 2.2 is an immediate consequence. 


\section{Acknowledgments}

I would like to thank K. Fieseler for valuable discussions and the referees for useful remarks and suggested improvements.

\section{References}

[Ad] R. M. Adin, On face numbers of rational simplicial polytopes with symmetry, Adv. in Math. 115 (1995), 269-285.

[BBD] A. Beilinson, J. Bernstein, and P. Deligne, Faisceaux pervers, Astérisque 100 (1982), 5-171.

[BL] L. J. Billera and C. W. Lee, A proof of the sufficiency of McMullen's condition for $f$-vectors of simplicial convex polytopes, J. Combin. Theory Ser. A 31 (1981), 237-255.

[DL] J. Denef and F. Loeser, Weights of exponential sums, intersection cohomology, and Newton polyhedra, Invent. Math. 106 (1991), 275-294.

[Fi] K.-H. Fieseler, Rational intersection cohomology of projective toric varieties, J. Reine Angew. Math. 413 (1991), 88-98.

[Fu] W. Fulton, Introduction to Toric Varieties, Annals of Mathematical Studies, Vol. 131, Princeton University Press, Princeton, NJ, 1993.

[Ki] F. Kirwan, Intersection homology and torus actions, J. Amer. Math. Soc. 1 (1988), 385-400.

[McM] P. McMullen, The polytope algebra, Adv. in Math. 87 (1989), 76-130.

[Sa] M. Saito, Mixed Hodge modules, Publ. Res. Inst. Math. Sci. 26 (1990), 221-333.

[St1] R. Stanley, The number of faces of a simplicial convex polytope, Adv. in Math. 35 (1980), 236-238.

[St2] R. Stanley, On the number of faces of centrally symmetric simplicial polytopes, Graphs Combin. 3 (1987), 55-66.

[St3] R. Stanley, Generalized $h$-vectors, intersection cohomology of toric varieties, and related results, in: Commutative Algebra and Combinatorics (M. Nagata and H. Matsumura, eds.), Advanced Studies in Pure Mathematics, Vol. 11, Kinokuniya, Tokyo, and North-Holland, Amsterdam, 1987, pp. 187-213.

[St4] R. Stanley, Combinatorics and Commutative Algebra, 2nd edn., Progress in Mathematics, Vol. 41, Birkhäuser, Boston, 1996.

Received November 6, 1997, and in revised form March 17, 1998. 